\title{
Apple Can Act as Anti-Aging on Yeast Cells
}

\author{
Vanessa Palermo, ${ }^{1}$ Fulvio Mattivi, ${ }^{2}$ Romano Silvestri, ${ }^{3}$ Giuseppe La Regina, ${ }^{3}$ \\ Claudio Falcone, ${ }^{1}$ and Cristina Mazzoni ${ }^{1}$ \\ ${ }^{1}$ Dipartimento di Biologia e Biotecnologie Charles Darwin, Istituto Pasteur-Fondazione Cenci Bolognetti, \\ Sapienza Università di Roma, Piazzale Aldo Moro 5, 00185 Roma, Italy \\ ${ }^{2}$ Food Quality and Nutrition Department, Research and Innovation Centre, Fondazione Edmund Mach, Via E. Mach 1, \\ 38010 San Michele all'Adige, Italy \\ ${ }^{3}$ Dipartimento di Chimica e Tecnologie del Farmaco, Istituto Pasteur-Fondazione Cenci Bolognetti, \\ Sapienza Università di Roma, Piazzale Aldo Moro 5, 00185 Roma, Italy
}

Correspondence should be addressed to Cristina Mazzoni, cristina.mazzoni@uniroma1.it

Received 31 May 2012; Revised 7 July 2012; Accepted 14 July 2012

Academic Editor: Paula Ludovico

Copyright (C) 2012 Vanessa Palermo et al. This is an open access article distributed under the Creative Commons Attribution License, which permits unrestricted use, distribution, and reproduction in any medium, provided the original work is properly cited.

In recent years, epidemiological and biochemical studies have shown that eating apples is associated with reduction of occurrence of cancer, degenerative, and cardiovascular diseases. This association is often attributed to the presence of antioxidants such as ascorbic acid (vitamin C) and polyphenols. The substances that hinder the presence of free radicals are also able to protect cells from aging. In our laboratory we used yeast, a unicellular eukaryotic organism, to determine in vivo efficacy of entire apples and their components, such as flesh, skin and polyphenolic fraction, to influence aging and oxidative stress. Our results indicate that all the apple components increase lifespan, with the best result given by the whole fruit, indicating a cooperative role of all apple components.

\section{Introduction}

During the last years, consumption of fruits and vegetables has received growing interest because many epidemiological and biochemical studies have demonstrated that they possess beneficial effects on human health. It is assumed that the cultivation of the apple tree goes back to prehistoric times and has developed with the formation of the $M . \times$ domestica gene pool from $M$. sieversii, originary of Thien Shan region, in Central Asia [1].

Apples can be eaten in countless ways, such as fresh and as juice that can be processed into cider, apple vinegar, or distilled.

Apples also contain nutrients and other compounds of interest, including high levels of polyphenols [2]. These compounds have biological activity and they can influence different mechanisms of fundamental relevance in cancer prevention [3]. The peel also contains high levels of triterpenoids, whereas red apples contain anthocyanins $[4,5]$.
The most studied molecules of this fruit are phenolic compounds that are distributed in the skin, in the core, and in the flesh. The composition of these compounds is quite variable depending on the environment of growth, period, and year of harvest. Generally, the average total polyphenol content of the most commonly consumed apples varies between $0.66 \mathrm{~g} / \mathrm{kg}$ and $2.1 \mathrm{~g} / \mathrm{kg}$ of fresh weight [6]. Apple polyphenols, and in particular the oligomeric proanthocyanidins, give the largest contribution to the antioxidant activity of apple extracts [7].

Numerous studies undertaken in recent years have shown that apples and their derivatives may have a wide range of biological activities, particularly useful in cases of asthma, cardiovascular disorders, polmonar disfunction and cancer. Studies in human models have shown that apples extracts may have a role in preventing several types of cancer. It has also been amply demonstrated beneficial effects on aging in mammalian skin [8]. 
Apple (sp. Malus domestica), is also rich in minerals, vitamin $\mathrm{B}$, citric, and malic acid that can contribute to the welfare of the body, promoting digestion and maintaining the acidity of the digestive system.

It has been proposed that anticancer action of apple is due to the presence of vitamin $\mathrm{C}$ and pectin, which, during its fermentation, produce butyric acid, a substance used in some experimental drugs to treat cancer [9].

It was also shown that polyphenolic-rich apple extracts may inhibit the activity of cytosolic PKC and have a role in the suppression of human cancer cell growth in vitro [8] indicating that apple's polyphenols could be of interest in cancer prevention $[2,3]$.

Yeast has been extensively used as a model organism for the study of complex phenomena that occur in higher eukaryotes. The use of yeast compared to mammalian cell culture, lies in the ease of manipulation and the large degree of homology between yeast and human genes.

Yeast is also used as a model to study aging as the principal pathways are conserved during evolution [10]. In recent years, several homologues of classical cell-death regulators have also been identified and characterized and yeast mutants that show an accelerated/delayed aging associated with early/late cell death are available to study the effect of molecules [11-13] on cell proliferation.

To test the effect of a substance on aging, cells are maintained in the presence of the substance to be tested and their ability to form colonies over the time is measured.

An increase in cell viability in cultures containing a substance, compared to the control, is a clear indicator of the effectiveness of such substance on the aging process.

To make a more sensitive analysis, in this work we used a peculiar mutant $(K l l s m 4 \Delta)$ that shows premature aging and cell death [14-16]. We cultivated such strain in the presence of apple extracts from skin, flesh, the entire fruit and the partially purified polyphenols fraction and we found that all of these components were able to increase lifespan at different extents, with the best results given by the whole fruit, indicating that the latter contains additional important substances. In view of future practical applications, we also verified the effectiveness of apple solutions included in ointment, pointing to this kind of preparation as a base for the prevention of skin aging.

\section{Results}

2.1. The Antiaging Power of Apples. To better understand the antiaging effects of apples, we followed the chronological aging of a Saccharomyces cerevisiae mutant that shows a very premature aging [16] in the presence of apple raw extracts from the entire fruit, flesh, skin and also in the presence of a purified polyphenols fraction. This mutant, named Kllsm $4 \Delta$, is mutated in the LSM4, a key gene of mRNA degradation, and shows a marked stabilization of mRNA accompanied by rapid loss of viability during aging $[14,17,18]$.

The comparison of the viability of the cells treated with different apple's extracts with the control cultures, can furnish a clear measure of their effectiveness on the aging process.

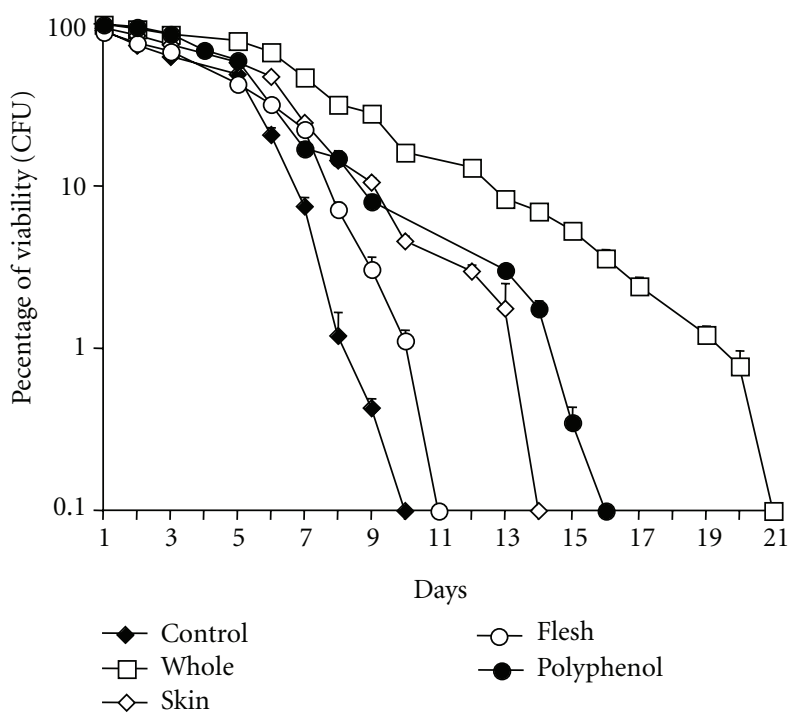

Figure 1: Apple components can recover premature aging of a yeast early-aging mutant. Cells of strain MCY4/Kllsm4::KanMX4 were grown in synthetic dextrose medium (SD) in the presence of extracts from whole apple, skin, flesh, and polyphenolic fractions. Acetone $7 \%$ was used as a control. Viability was monitored as percentage of microcolony forming units (CFU). Standard deviation was obtained from three independent experiments.

Apple's extracts consisting of freshly prepared, centrifuged and sterile filtered apple juice resuspended into $70 \%$ acetone (See Section 4 ) were added, prior ten time dilution, to Kllsm $4 \Delta 1$ cultures at the concentration of about $26 \mathrm{mg} / \mathrm{mL}$, and the chronological aging during stationary phase of yeast samples was followed for 21 days.

As shown in Figure 1, the death kinetics of the Kllsm $4 \Delta 1$ strain grown in synthetic medium containing only $7 \%$ acetone is much faster compared to the cultures containing apple extracts. This difference, clearly evident since the early days, increases with time and we observed the complete loss of viability of control cultures after ten days of treatment.

In contrast, cultures containing the extracts from flesh and peel completely lost viability after 11 and 14 days, respectively, while in the presence of the whole apple extract viability ceased after twenty-one days, with an extension of more than $100 \%$ compared to control cultures. Also polyphenols extracts exerted a marked effect in prolonging lifespan, conferring an extension of viability of $60 \%$.

It has been suggested that reactive oxygen species (ROS) are the main cause of cellular aging as they can damage and cross-link DNA, proteins, and lipids.

For this reason, we looked by dihydrorhodamine (DHR) 123 staining at the accumulation of ROS in yeast exponential and stationary (aged) cells grown in the presence of apple components.

As previously demonstrated [19] and shown in Figure 2, DHR staining revealed that in the absence of external oxidative stress, Kllsm $4 \Delta 1$ cells accumulated ROS during exponential phase (a) which increased with aging (b). The presence of apple components prevented ROS accumulation 
in both exponential and stationary phase cells, with the exception of the apple flesh.

This component, unexpectedly, seems to increase the production of ROS since the percentage of cells showing ROS was higher than in control cultures both in exponential and stationary phase (Figure 2(c)).

We then checked the effect of apple extracts on cell survival following an oxidative stress such as the treatment of cultures with hydrogen peroxide. The presence of flesh or skin extracts could not protect cells from such a stress in that viability was comparable to that of control cultures (Figure 3), and, at the concentration of $0,8 \mathrm{M} \mathrm{H}_{2} \mathrm{O}_{2}$, viability was unclearly even lower.

On the contrary, both the polyphenols and the whole extracts conferred to the cells higher resistance to hydrogen peroxide treatment. As shown in Figure 3, at the highest $\mathrm{H}_{2} \mathrm{O}_{2}$ concentration tested $(3 \mathrm{mM})$ cells of control cultures showed a viability of $15 \%$, while in the presence of whole apple and polyphenolic extracts showed a viability of $28 \%$ and $20 \%$, respectively, suggesting that these extracts contained antioxidant activity having an important role in preventing damage from oxidative stress.

Then we wanted to confirm the results obtained with the purified extracts using formulation containing a solution from homogenates of flesh, skin, or the whole apple added to ointment base. This kind of ointment might represent the basis for a cosmetic use of apple for skin care.

We first evaluated the chronological aging of $10 \mathrm{~mL}$ yeast cultures grown in the presence of 1 gr of formulation containing the apple solutions. As can be seen in Figure 4(a), compared to the control, flesh and peel creams extended lifespan of about $7 \%$ and $28,5 \%$, respectively. The most striking result was obtained with the whole apple homogenate that extended cell viability of about $78,5 \%$.

We also performed an additional test (viability spot assay) based on serial dilutions of treated cultures followed by cell plating on rich medium containing glucose as carbon source. We incubated the plates at $28^{\circ} \mathrm{C}$ and we then followed the growth of strain Kllsm $4 \Delta 1$ for 15 days on plates containing rich medium (YPD) at intervals of three days. As shown in Figure 4 (b) after 20 days of growth at $28^{\circ} \mathrm{C}$, only the culture containing the whole apple survived better until the third dilution, confirming the data on chronological aging.

We also evaluated the capacity of the creams containing the different apple components to contrast the oxidative stress induced by treatment with hydrogen peroxide.

As shown in Figure 4(c), the cream added with the whole apple solution showed the best protective effect against oxidative stress in the presence of $0.8 \mathrm{mM} \mathrm{H}_{2} \mathrm{O}_{2}$ while, at increasing concentrations the compound, cell grown with whole apple and skin containing creams showed the highest protection.

The apple extracts, which were prepared containing a similar concentration of apple (see Section 4), clearly differ in their concentration of total polyphenols, ranging from a minimum (ca. $138 \mathrm{mg} / \mathrm{l}$ ) in apple flesh and in the partially purified polyphenols, to intermediate values $(189 \mathrm{mg} / \mathrm{l})$ in the whole apple extract, up to much higher concentration $(584 \mathrm{mg} / \mathrm{l})$ in the apple skin extract. In conclusion, the total polyphenols ranged in our extracts as follows: skin $\gg$ whole apple $>$ flesh.

The apple extracts were screened also with an untargeted LCMS method, in order to provide an unbiased, semiquantitative fingerprint of their composition. These data can be useful to estimate the relative concentration of known compounds, as well as for extracting additional information useful for the design of future metabolomics experiment. The major apple phenolics according to the literature [6] were assessed and their presence in the extracts is reported in Figure 5. The extracts were screened for the presence of the most representative flavanols (catechin and epicatechin), procyanidin (procyanidin B2), dihydrochalcones (phloridzin), cynnamic acids (chlorogenic acid), and flavonols (quercetin glycosides).

The qualitative composition of these extracts was expected to vary in light of the specific localization of apple polyphenols in the different tissues [20]. In Golden Delicious apple the quercetin glycosides, and in particular the quercetin hexosides, are more concentrated in the skin cell layers lying just below the cuticle, while in the pericarp they are located mainly in correspondence with the vascular system, and the core is relatively rich in quercetinrhamnoside [21]. Once the not edible part (the core) is removed, the apple flesh is almost devoid of flavonols (Figure 5). Also the procyanidins B2, are more concentrated in the cell layers, ca. 150 micron thick, just below the cuticle while its concentration decreases inside the fruit [21] and this is reflected in the composition of the extract in flavonols (Figure 5).

In summary and in agreement with the literature, apple flavonols were almost absent in the flesh extract, and ca. 3 times more concentrated in skin extract in respect of both whole apple and purified polyphenols extracts. The major flavonols (epicatechin and procyanidin B2), and phloridzin were 2 times higher in skin versus the other extracts. Both catechin and chlorogenic acid were contained in similar concentrations in all the extracts, including the flesh.

\section{Discussion}

Besides health care, more and more products prolonging youth and acting against aging come to market each year. For most of them, clear scientific demonstration of effectiveness is insufficient or even absent.

In vivo tests with cell cultures are expensive, as well those with animals that, in addition, are rejected by an increasing part of the society for ethical aspects.

For many years yeast has been used as a simple model for the study of basic mechanism underlying the complex phenomena occurring in higher eukaryotes.

Among many other advantages, the knowledge of the entire genome and the availability of mutants in each of the near six thousand genes render this organism a powerful tool for basal as well as for applicative studies.

In this respect, we successfully used yeast to evaluate the toxicity of new arylthioindoles (ATIs), which are potent tubulin assembly inhibitors [22, 23]. 


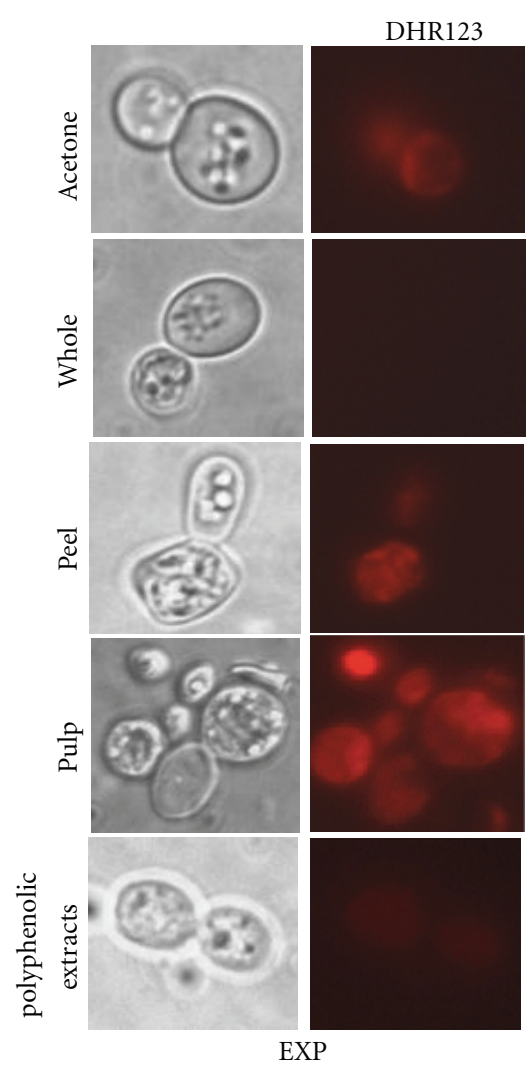

(a)

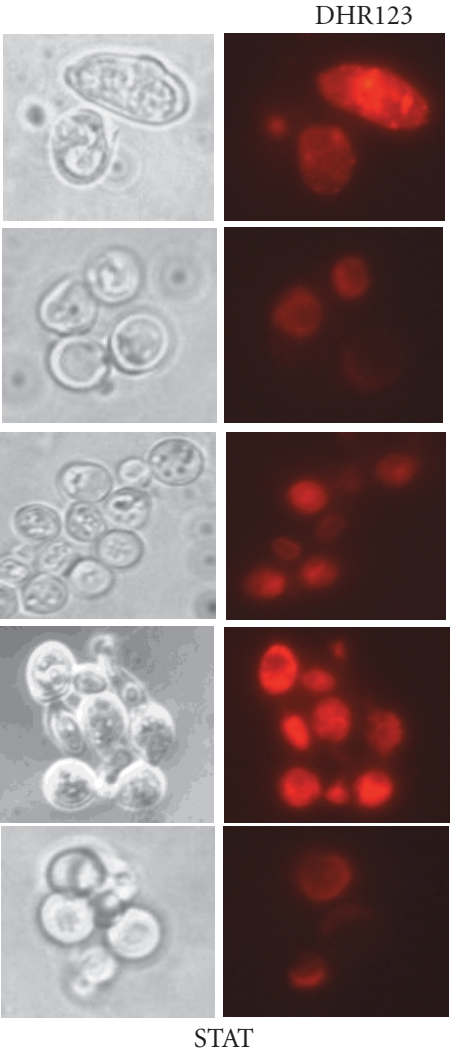

(b)

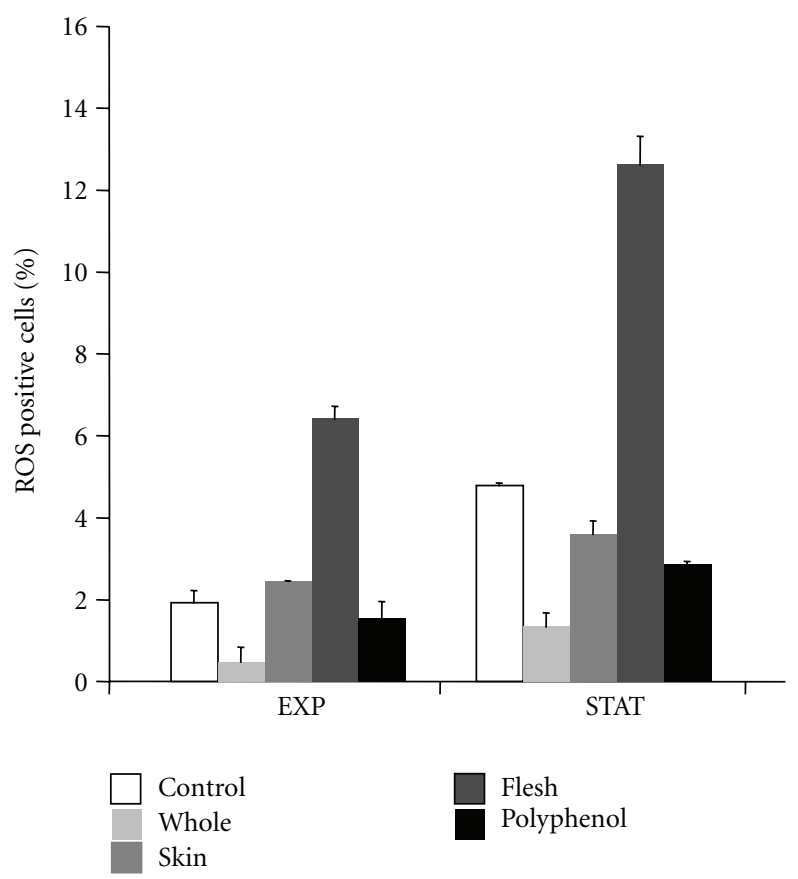

(c)

FIGURE 2: Effect of apple components on the production of intracellular ROS during aging. MCY4/Kllsm4::KanMX4 yeast cells were grown in the presence of extracts from the peel, flesh, or entire apple and the polyphenolic fraction. Acetone was used as a control. Cells from exponential (EXP; (a)) and stationary (STAT; (b)) cultures were incubated with DHR123 and after $3 \mathrm{~h}$ observed at the fluorescence microscope. In (c) is reported the percentage of ROS positive cells. Average and standard deviations, obtained from three independent experiments, are also indicated. 


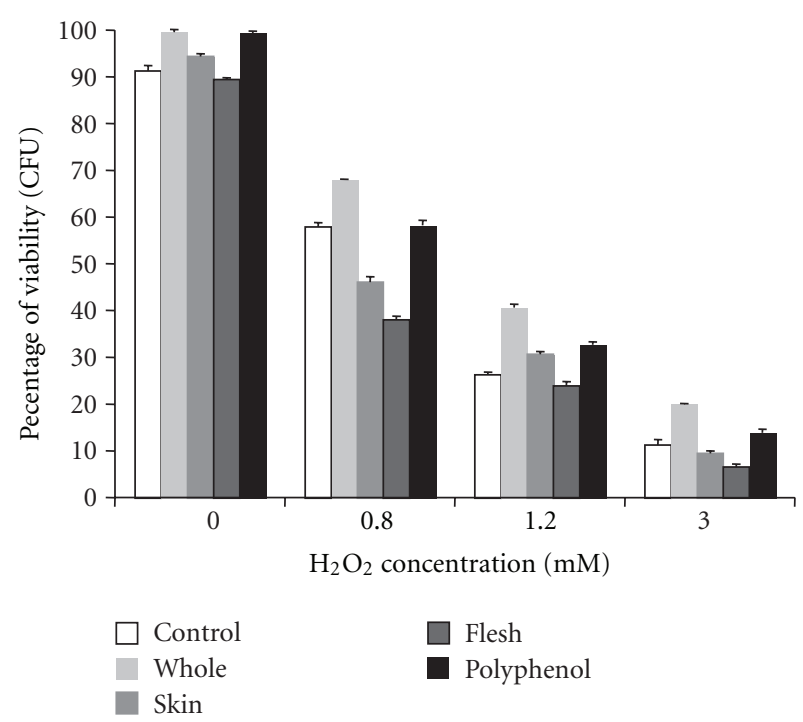

FIGURE 3: Effect of apple components on the oxidative stress resistance. Viability of the MCY4/Kllsm $4 \Delta 1$ strain was measured after exposure of cells to $\mathrm{H}_{2} \mathrm{O}_{2}$ at the indicated concentration for $4 \mathrm{~h}$ in the presence of acetone (control) or extracts from whole apple, skin, flesh, and polyphenolic fractions. Average and standard deviations, obtained from three independent experiments, are indicated.

In another work, by the use of a particular strain of $S$. cerevisiae, mutated in the LSM4 gene and showing premature aging and loss of viability, we demonstrated that carnitines, in particular acetyl-L-carnitine (ALC), are able to prolong lifespan, to protect cells from oxidative stress and prevent mitochondrial fragmentation [13].

LSM4 is an essential gene encoding a subunit of the Lsm complex involved in mRNA decapping and splicing [17]. In previous works we demonstrated that S. cerevisiae strains not expressing the endogenous Lsm4p recovered viability when transformed with Kllsm4, the hortolog gene from Kluyveromyces lactis, as well with a truncated form of this gene $(K l l s m 4 \Delta 1)$ still containing the Sm-like domains The Kllsm $4 \Delta 1$ mutants showed accumulation of mRNA degradation intermediates, the increase of intracellular ROS, undergo cell death prematurely during stationary phase and show the typical markers of apoptosis. A quite normal cell viability observed in one of these mutants (lsm4) was recovered following the deletion of the yeastmeta-caspase YCA1 gene, suggesting that caspase activity is required for apoptosis induced by increased mRNA stability [16, 24, 25].

The short lifespan and the high sensitivity to oxidative stress of Kllsm4 1 mutants, compared to wild-type yeast strains, render them good candidates to test the ability of substances to prevent aging and cell death.

In this paper, we used this particular mutant to study the capability of apple's solution and derived fractions to increase viability and oxidative stress resistance in yeast cells.

Our result show that solutions of entire apples can prolong cellular life span by $100 \%$, more than any single apple fractions. In fact, while flesh showed a modest effect, skin increased life span by $40 \%$, probably due to its content of antioxidant polyphenols, as also suggested by the $60 \%$ increase of viability observed with cultures treated with purified polyphenol fractions. The fact that the purified polyphenols had a quite high activity, speaks in favour of an important role of apple polyphenols. However, since the whole apple extract had superior activity in spite of a much lower content of polyphenols in respect of the skin extract suggested that compounds other than polyphenols are contributing to the observed extension of the cellular life span.

Similarly, more than skin and polyphenols, entire apples strongly reduced ROS production in both exponential and stationary aging cells. In contrast, apple flesh strongly increased ROS production, especially in stationary phase cells.

Since flesh shows a very low positive effect on cell viability, one can conclude that the potential toxicity of the observed increase of ROS is in some way neutralized by other components present in the entire apple.

Whole apple extracts, closely followed by the polyphenol fraction, can also protect cells efficiently from hydrogen peroxide treatments while skin and pulp have a minor effect slightly varying with $\mathrm{H}_{2} \mathrm{O}_{2}$ concentration.

These results, in their substance, were confirmed when the same fractions were included into a creamy matrix, with the entire apple homogenate showing the best protection.

These findings are in agreement with Vanzani et al. [7] who reported that the antioxidant efficiency of the pure apple compounds was lower than that of the apple extracts, and that the higher efficiency of apples appears to be strictly related to the overwhelming presence of oligomeric proanthocyanidins.

In conclusion, the wide knowledge of yeast and the availability of genetic, biochemical and molecular biology tools make this organism a simple system useful for assaying in vivo the effects, among others, of anti-aging molecules. The promising results of this preliminary study provide the basis to plan further researches aimed to precisely identifying the apple compounds capable to modulate the life-extending properties of apple on yeasts.

\section{Materials and Methods}

4.1. Yeast Strains and Growth Conditions. We used the S. cerevisiae strains MCY4/KILSM4::kanMX4 (MAT $\alpha$, ade1101, his3- 1 1, trp1-289, ura3, LEU-GAL1-SDB23, pRS416/

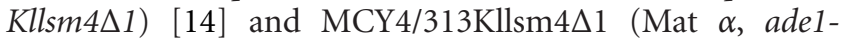
101, his3- 1 1, trp1-289, ura3, LEU2-GAL1-SDB23, pRS313/ Kllsm4 1 ) [16].

Cells were grown in YP (1\% yeast extract, $2 \%$ peptone) supplemented with $2 \%$ glucose (YPD) at $28^{\circ} \mathrm{C}$ with the addition of or in SD (yeast nitrogen base without amino acids and auxotrophic requirement as needed). Solid media were supplemented with $2 \%$ Bactoagar (Difco, Detroit, MI, USA).

4.2. Cell Viability. The determination of chronological lifespan was done as described in Palermo et al., 2007 [26]. Briefly, cell suspensions $(5 \mu \mathrm{L})$ containing approximately 


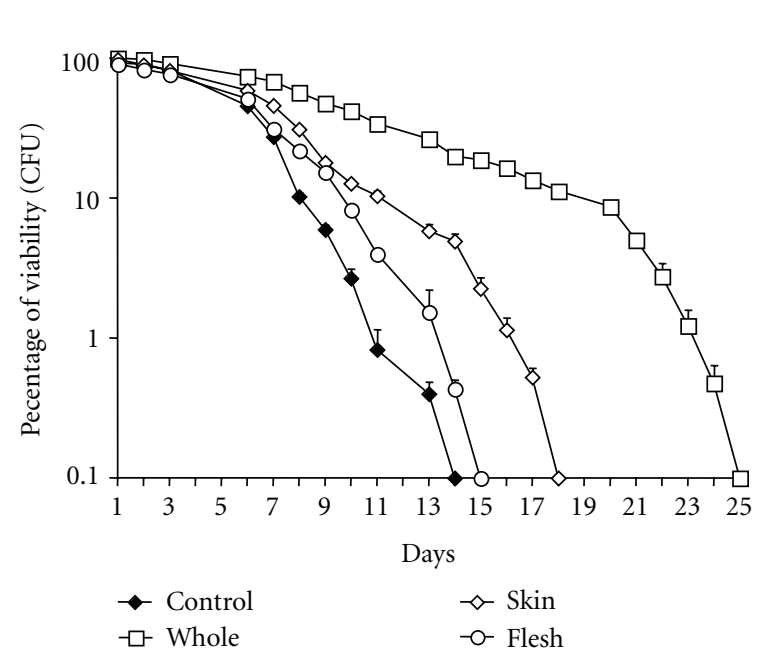

(a)
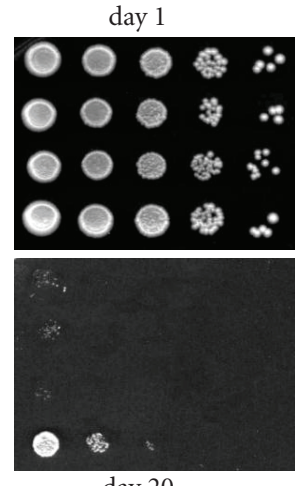

day 20

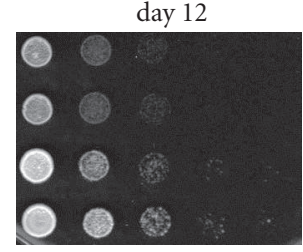

Control

Flesh

Skin

Whole

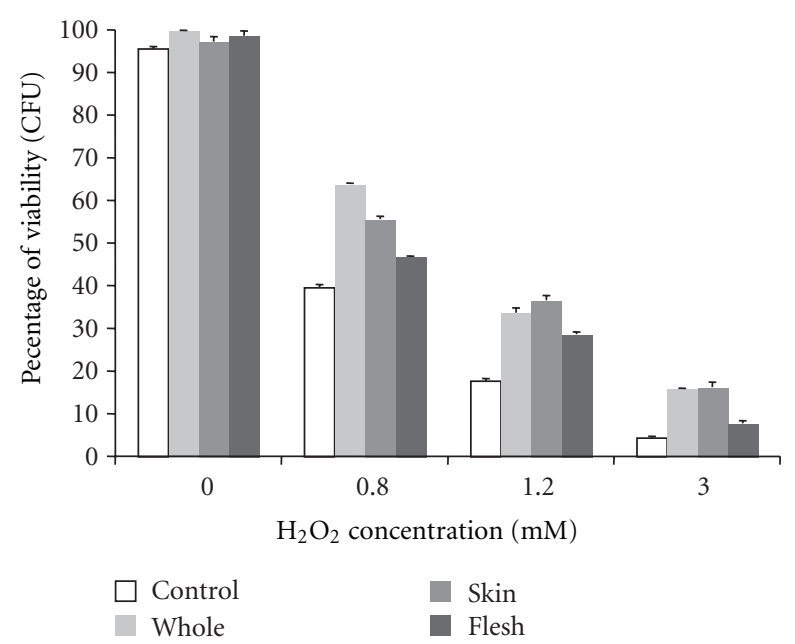

(b)

(c)

FIGURE 4: Effect of apple solutions included in ointment. (a) Cells of strain MCY4/Kllsm4::KanMX4 were grown in YPD+G418 antibiotic to prevent contaminations. Ointment base Essex was used as a control. Viability was monitored as percentage of microcolony forming units. Standard deviation was obtained from three independent experiments. (b) 10-fold serial dilutions of each cell culture described above were spotted onto YPD plates (1\% bactopeptone, $1 \%$ yeast extract, $2 \%$ glucose, and $2 \%$ bacto agar) and incubated 2 days before recording. (c) Cells of strain MCY4/Kllsm4::KanMX4 were grown in YPD + G418 antibiotic in the presence of apple solutions in ointment and exposed to $\mathrm{H}_{2} \mathrm{O}_{2}$ at the indicated concentration for $4 \mathrm{~h}$. Average and standard deviations, obtained from three independent experiments are indicated.

$6 \cdot 10^{6}$ cells $\mathrm{mL}^{-1}$ were poured on a thin layer of YPD agar on a microscope slide. A cover slip was placed over the samples and, after $24 \mathrm{~h}$, viable and unviable cells were scored on the basis of their ability to form microcolonies.

Cells were grown at $28^{\circ} \mathrm{C}$ in minimal medium $(0,67 \%$ yeast nitrogen base without amino acids) containing $2 \%$ glucose (SD) supplemented with $20 \mathrm{microg} / \mathrm{mL}$ of the appropriate nutritional requirements according to the genotype of the strains, or in YPD + G418.

For the viability spot assay, cell suspensions at the concentration of $10^{7}$ cells $/ \mathrm{mL}$ were transferred in microtiter plates, serially ten-fold diluted and spotted onto YP plates ( $1 \%$ yeast extract, $2 \%$ peptone) supplemented with $2 \%$ glucose (YPD). Plates were incubated at $28^{\circ} \mathrm{C}$ for five days before recording.

4.3. $\mathrm{H}_{2} \mathrm{O}_{2}$ Sensitivity. To determine the sensitivity to oxygen peroxide, cells growing exponentially were exposed to 0.8 , 1.2 , and $3 \mathrm{mM} \mathrm{H}_{2} \mathrm{O}_{2}$ at $28^{\circ} \mathrm{C}$ for $4 \mathrm{~h}$. Cell viability was determined by counting the formation of microcolonies.

4.4. ROS Detection. Dihydrorhodamine 123 (Sigma) was added at a concentration of $5 \mu \mathrm{g} / \mathrm{mL}$ of cell culture from a $2.5 \mathrm{mg} / \mathrm{mL}$ stock solution in ethanol and cells were viewed without further processing through a rhodamine optical filter after $3 \mathrm{~h}$ incubation [27]. 


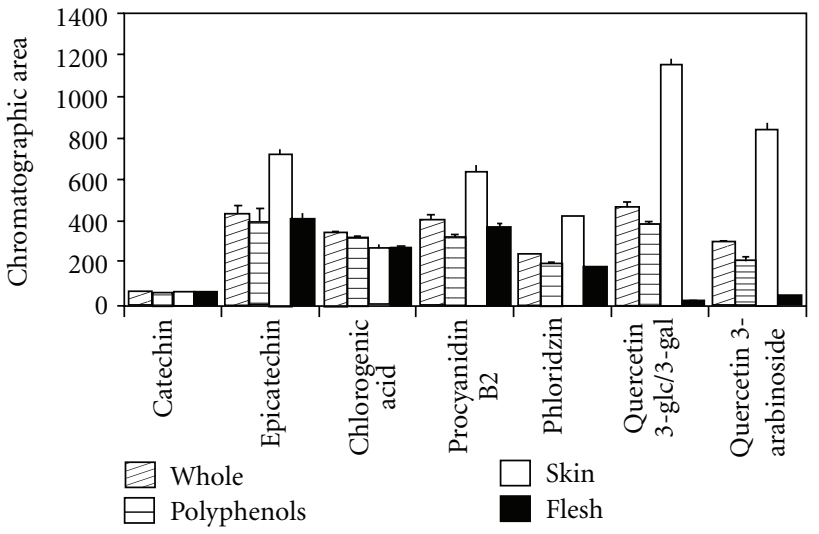

FIGURE 5: Comparison of the average concentrations (expressed as chromatographic area) of some characteristic apple polyphenols in the extracts used for the viability tests. The vertical bars represent the standard deviation of the measure.

\subsection{Sample Preparation}

Extracts. Apples, from the cv. Golden Delicious, produced in Trentino, Italy, were purchased directly from the producers in June 2009, and extracted as described in Vrhovsek [6]. To limit the enzymatic oxidation and chemical reactions, both the apples and the extraction solution were cooled to $4{ }^{\circ} \mathrm{C}$. Each sample was prepared out of three fruits. The core was removed with a corer, in order to study only the edible part.

Whole Apple Extract. Each apple was cut into equal slices. Three slices (cortex + skin) from the opposite side of each fruit (total weight $400 \mathrm{~g}$ ) were used for the preparation of aqueous acetone extracts. The sample was homogenized in a blender Osterizer mod. 847-86 at speed one in $1500 \mathrm{~mL}$ of mixture acetone/water $(70 / 30 \mathrm{w} / \mathrm{w})$, to produce a raw extract corresponding to $26.7 \mathrm{~g}$ of fresh apple in $100 \mathrm{~mL}$.

Whole Apple Polyphenols. An aliquot of $750 \mathrm{~mL}$ of the above extract, corresponding to $200 \mathrm{~g}$ of apple, was used to prepare a partially purified extract, containing most apple polyphenols. Acetone was removed by evaporation under reduced pressure at $35^{\circ} \mathrm{C}$, from a volume of $200 \mathrm{~mL}$ of apple polyphenols extract, which was brought to $100 \mathrm{~mL}$ with water. This extract was loaded on a preparative column containing $20 \mathrm{~g}$ of Isolute ENV + resin (Biotage, Uppsala, Sweden), previously activated with $300 \mathrm{~mL} \mathrm{MeOH}$ and $400 \mathrm{~mL}$ water. All the hydrophilic compounds were washed with $300 \mathrm{~mL}$ of water, then the polyphenols were eluted with $400 \mathrm{~mL}$ of methanol. After the whole extract was processed, the methanol was removed by evaporation under reduced pressure, and the purified fraction was redissolved into $750 \mathrm{~mL}$ of acetone/water $(70 / 30 \mathrm{w} / \mathrm{w})$.

Skin Extract. Each apple was manually peeled. An aliquot of $40 \mathrm{~g}$ of apple skins was extracted with $166 \mathrm{~mL}$ of the mixture acetone/water $(70 / 30 \mathrm{w} / \mathrm{w})$, to produce a raw extract corresponding to $24.0 \mathrm{~g}$ of apple skins in $100 \mathrm{~mL}$.
Flesh Extract. Each apple was manually peeled. An aliquot of $200 \mathrm{~g}$ of apple flesh (cortex) was extracted with $750 \mathrm{~mL}$ of the mixture acetone/water $(70 / 30 \mathrm{w} / \mathrm{w})$, to produce a raw extract corresponding to $26.7 \mathrm{~g}$ of apple cortex in $100 \mathrm{~mL}$.

The centrifuged extracts were stored at $-20^{\circ} \mathrm{C}$ for the cell viability trials and analysis of total polyphenols.

Ointments. Fresh apples were crushed and filtered. The clear liquid $(20 \mathrm{~mL})$ was added to ointment base Essex $(80 \mathrm{~g})$ while stirring.

\subsection{Chemical Analyses}

Spectrophotometric Analysis of Total Polyphenols. The total amount of polyphenols was quantified with an optimized Folin-Ciocalteu method [28] according to which interfering compounds such as sugars, amino acids, and ascorbic acid were removed by cleanup on a C-18 cartridge $(0.5 \mathrm{~g}$, Sep pak, Waters) from the sample reconstituted in water as described above. The results are expressed as equivalent of (+)-catechin, $\mathrm{mg} / \mathrm{kg}$ of FW.

Fingerprinting of the Extracts by LCMS. Analysis was carried out using a Waters Acquity UPLC, coupled to a Synapt HDMS QTOF-MS (Waters, Manchester, UK) via an electrospray interface (ESI), operating in $\mathrm{W}$-mode. The software used was Masslynx 4.1. The reverse phase method was performed on a ACQUITY UPLC $1.8 \mu \mathrm{m} 2.1 \times 150 \mathrm{~mm}$ HSS T3 (Waters) column, maintained at $30^{\circ} \mathrm{C}$ for $30 \mathrm{~min}$ using $0.1 \%$ formic acid in water as solvent $\mathrm{A}$ and $0.1 \%$ formic acid in methanol as solvent $\mathrm{B}$ with the following gradient: until 6 min isocratic at $100 \% \mathrm{~A}$, then increasing linearly to $100 \% \mathrm{~B}$ at $26 \mathrm{~min}$ and held isocratic at $100 \% \mathrm{~B}$ till $30 \mathrm{~min}$. After each run, the column was brought the initial conditions $(30 \%$ of solvent A) in $3 \mathrm{~min}$.

Spectra were collected in negative ESI mode over a mass range 50-3000 amu with a scan duration of $0.3 \mathrm{~s}$ in centroid mode. The transfer collision energy and trap collision energy were set at 4 and $6 \mathrm{~V}$, respectively. The source parameters were: capillary $2.5 \mathrm{kV}$, sampling cone $25 \mathrm{~V}$, extraction cone $3 \mathrm{~V}$, source temperature $150^{\circ} \mathrm{C}$, desolvation temperature $500^{\circ} \mathrm{C}$, desolvation gas flow $1000 \mathrm{l} / \mathrm{h}$, and nebulizer gas flow $50 \mathrm{l} / \mathrm{h}$. External calibration of the instrument was performed at the beginning of each batch of analysis by direct infusion of a sodium formate solution ( $10 \%$ formic acid/ $0.1 \mathrm{M}$ $\mathrm{NaOH} /$ Acetonitrile at a ratio of $1 / 1 / 8$ ) by controlling the mass accuracy (less than $5 \mathrm{ppm}$ ) and mass resolution (over 14000 FWHM). LockMass calibration was applied using a solution of leucine enkephaline $(0.5 \mathrm{mg} / \mathrm{L}, \mathrm{m} / z 556.2771$ for positive and 554.2620 for negative ion mode) at $0.1 \mathrm{~mL} / \mathrm{min}$.

Each extract was analysed 10 times. The relative concentration of major apple polyphenols in the extracts are given in Figure 5.

\section{Acknowledgments}

Domenico Masuero is acknowledged for excellent technical 
support in the spectrophotometric and LCMS analysis. This work was supported by PRIN 2009.

\section{References}

[1] R. Velasco, A. Zharkikh, J. Affourtit et al., "The genome of the domesticated apple (Malus $\times$ domestica Borkh.)," Nature Genetics, vol. 42, no. 10, pp. 833-839, 2010.

[2] S. Reagan-Shaw, D. Eggert, H. Mukhtar, and N. Ahmad, "Antiproliferative effects of apple peel extract against cancer cells," Nutrition and Cancer, vol. 62, no. 4, pp. 517-524, 2010.

[3] C. Gerhauser, "Cancer chemopreventive potential of apples, apple juice, and apple components," Planta Medica, vol. 74, no. 13, pp. 1608-1624, 2008.

[4] J. Boyer and R. H. Liu, "Apple phytochemicals and their health benefits," Nutrition Journal, vol. 3, article no. 1, pp. 1-45, 2004.

[5] T. K. McGhie, S. Hudault, R. C. M. Lunken, and J. T. Christeller, "Apple peels, from seven cultivars, have lipaseinhibitory activity and contain numerous ursenoic acids as identified by LC-ESI-QTOF-HRMS," Journal of Agricultural and Food Chemistry, vol. 60, no. 1, pp. 482-491, 2012.

[6] U. Vrhovsek, A. Rigo, D. Tonon, and F. Mattivi, "Quantitation of polyphenols in different apple varieties," Journal of Agricultural and Food Chemistry, vol. 52, no. 21, pp. 6532-6538, 2004.

[7] P. Vanzani, M. Rossetto, A. Rigo et al., "Major phytochemicals in apple cultivars: contribution to peroxyl radical trapping efficiency," Journal of Agricultural and Food Chemistry, vol. 53, no. 9, pp. 3377-3382, 2005.

[8] R. H. Liu and J. Sun, "Antiproliferative activity of apples is not due to phenolic-induced hydrogen peroxide formation," Journal of Agricultural and Food Chemistry, vol. 51, no. 6, pp. 1718-1723, 2003.

[9] M. Waldecker, T. Kautenburger, H. Daumann et al., "Histonedeacetylase inhibition and butyrate formation: fecal slurry incubations with apple pectin and apple juice extracts," Nutrition, vol. 24, no. 4, pp. 366-374, 2008.

[10] M. G. Mirisola and V. D. Longo, "Conserved role of Ras-GEFs in promoting aging: from yeast to mice," Aging, vol. 3, pp. 340343, 2011.

[11] D. Carmona-Gutierrez, T. Eisenberg, S. Büttner, C. Meisinger, G. Kroemer, and F. Madeo, "Apoptosis in yeast: triggers, pathways, subroutines," Cell Death and Differentiation, vol. 17, no. 5, pp. 763-773, 2010.

[12] A. Cassidy-Stone, J. E. Chipuk, E. Ingerman et al., "Chemical inhibition of the mitochondrial division dynamin reveals its role in bax/bak-dependent mitochondrial outer membrane permeabilization," Developmental Cell, vol. 14, no. 2, pp. 193204, 2008.

[13] V. Palermo, C. Falcone, M. Calvani, and C. Mazzoni, "AcetylL-carnitine protects yeast cells from apoptosis and aging and inhibits mitochondrial fission.," Aging cell, vol. 9, no. 4, pp. 570-579, 2010.

[14] C. Mazzoni and C. Falcone, "Isolation and study of KILSM4, a Kluyveromyces lactis gene homologous to the essential gene LSM4 of Saccharomyces cerevisiae," Yeast, vol. 18, no. 13, pp. 1249-1256, 2001.

[15] C. Mazzoni, P. Mancini, F. Madeo, V. Palermo, and C. Falcone, "A Kluyveromyces lactis mutant in the essential gene KILSM4 shows phenotypic markers of apoptosis," FEMS Yeast Research, vol. 4, no. 1, pp. 29-35, 2003.

[16] C. Mazzoni, E. Herker, V. Palermo et al., "Yeast caspase 1 links messenger RNA stability to apoptosis in yeast," EMBO Reports, vol. 6, no. 11, pp. 1076-1081, 2005.
[17] M. Cooper, L. H. Johnston, and J. D. Beggs, "Identification and characterization of Uss1p (Sdb23p): a novel U6 snRNAassociated protein with significant similarity to core proteins of small nuclear ribonucleoproteins," EMBO Journal, vol. 14, no. 9, pp. 2066-2075, 1995.

[18] A. E. Mayes, L. Verdone, P. Legrain, and J. D. Beggs, "Characterization of Sm-like proteins in yeast and their association with U6 snRNA," EMBO Journal, vol. 18, no. 15, pp. 43214331, 1999.

[19] C. Mazzoni, P. Mancini, L. Verdone et al., "A truncated form of K1Lsm4p and the absence of factors involved in mRNA decapping trigger apoptosis in yeast," Molecular Biology of the Cell, vol. 14, no. 2, pp. 721-729, 2003.

[20] F. Chinnici, A. Bendini, A. Gaiani, and C. Riponi, "Radical scavenging activities of peels and pulps from cv. golden delicious apples as related to their phenolic composition," Journal of Agricultural and Food Chemistry, vol. 52, no. 15, pp. 4684-4689, 2004.

[21] P. Franceschi, Y. Dong, K. Strupat, U. Vrhovsek, and F. Mattivi, "Combining intensity correlation analysis and MALDI imaging to study the distribution of flavonols and dihydrochalcones in Golden Delicious apples," Journal of Experimental Botany, vol. 63, no. 3, pp. 1123-1133, 2012.

[22] G. La Regina, T. Sarkar, R. Bai et al., "New arylthioindoles and related bioisosteres at the sulfur bridging group. 4. Synthesis, tubulin polymerization, cell growth inhibition, and molecular modeling studies," Journal of Medicinal Chemistry, vol. 52, no. 23, pp. 7512-7527, 2009.

[23] V. Palermo, L. Pieri, R. Silvestri, G. La Regina, C. Falcone, and C. Mazzoni, "Drug-induced inhibition of tubulin polymerization induces mitochondrion-mediated apoptosis in yeast," Cell Cycle, vol. 10, no. 18, pp. 3208-3209, 2011.

[24] C. Mazzoni and C. Falcone, "mRNA stability and control of cell proliferation," Biochemical Society Transactions, vol. 39, no. 5, pp. 1461-1465, 2011.

[25] C. Mazzoni and C. Falcone, "Caspase-dependent apoptosis in yeast," Biochimica et Biophysica Acta, vol. 1783, no. 7, pp. 1320-1327, 2008.

[26] V. Palermo, C. Falcone, and C. Mazzoni, "Apoptosis and aging in mitochondrial morphology mutants of S. cerevisiae," Folia Microbiologica, vol. 52, no. 5, pp. 479-483, 2007.

[27] F. Madeo, E. Fröhlich, M. Ligr et al., "Oxygen stress: a regulator of apoptosis in yeast," Journal of Cell Biology, vol. 145, no. 4, pp. 757-767, 1999.

[28] A. Rigo, F. Vianello, G. Clementi et al., "Contribution of proanthocyanidins to the peroxy radical scavenging capacity of some Italian red wines," Journal of Agricultural and Food Chemistry, vol. 48, no. 6, pp. 1996-2002, 2000. 


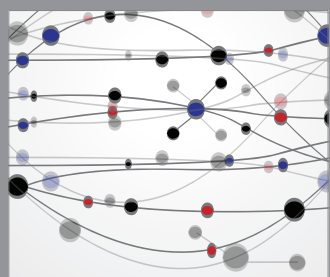

The Scientific World Journal
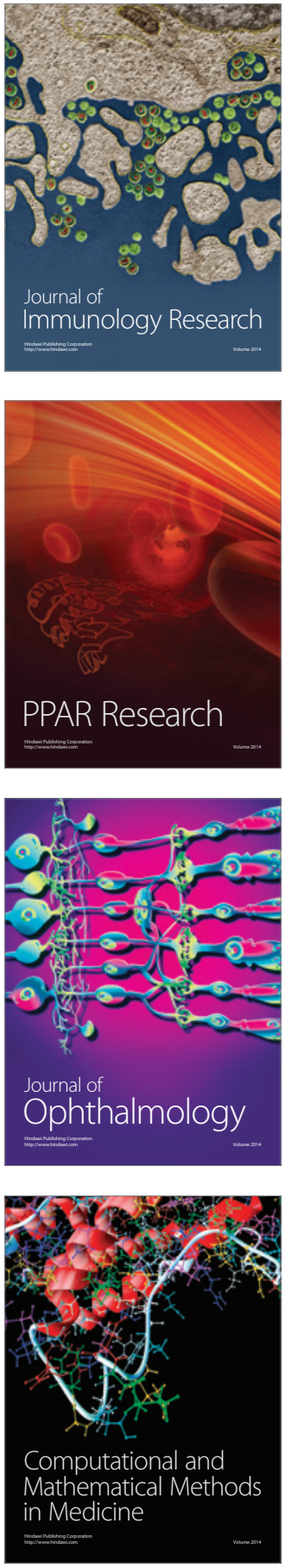

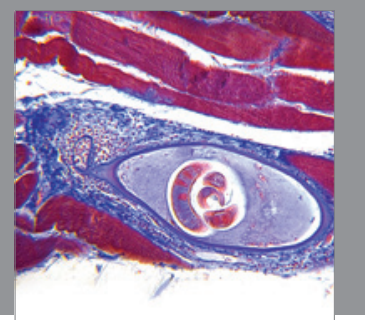

Gastroenterology

Research and Practice
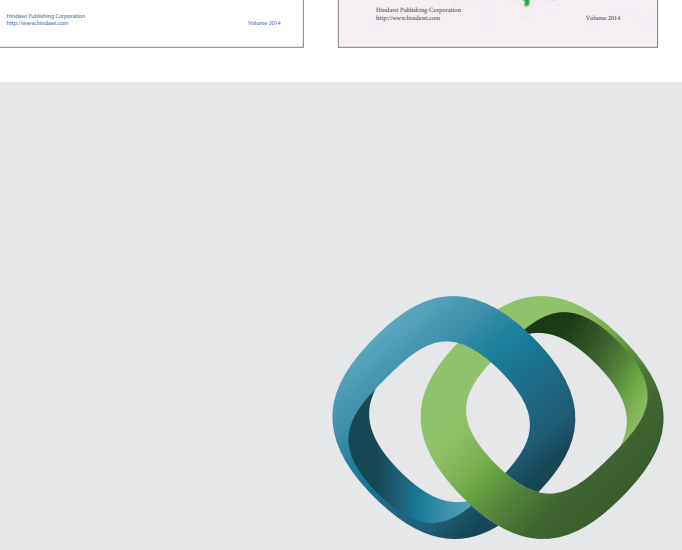

\section{Hindawi}

Submit your manuscripts at

http://www.hindawi.com
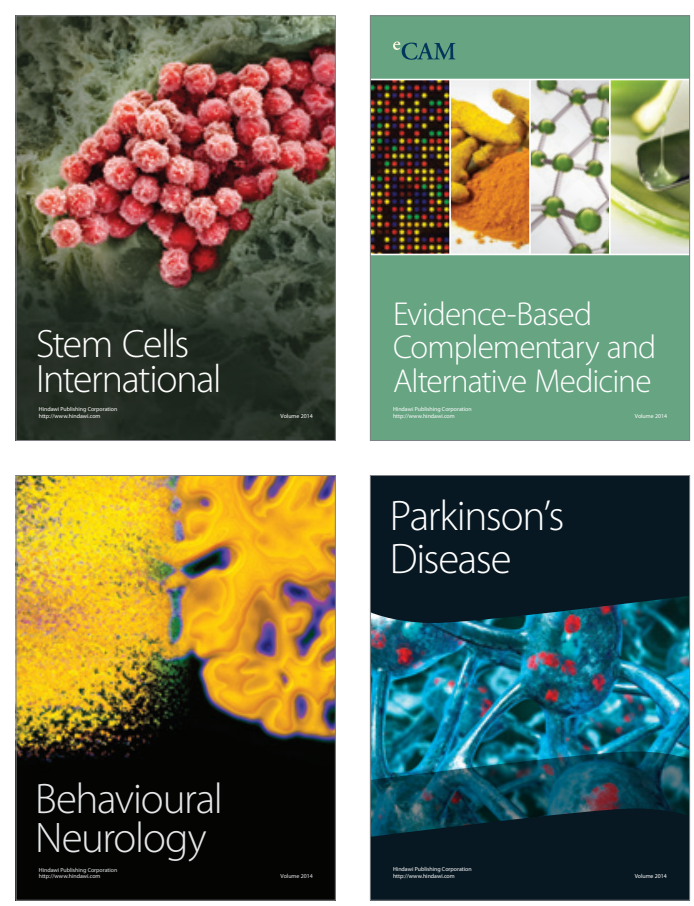

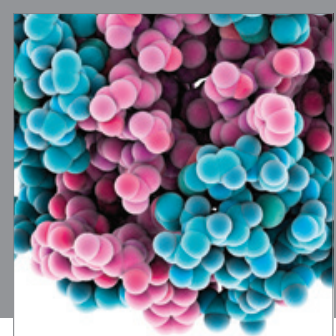

Journal of
Diabetes Research

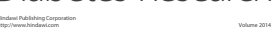

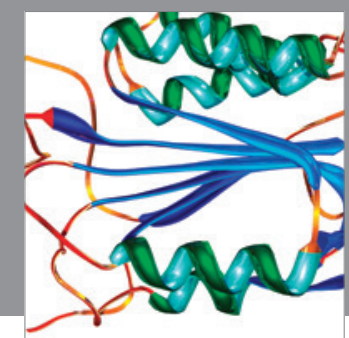

Disease Markers
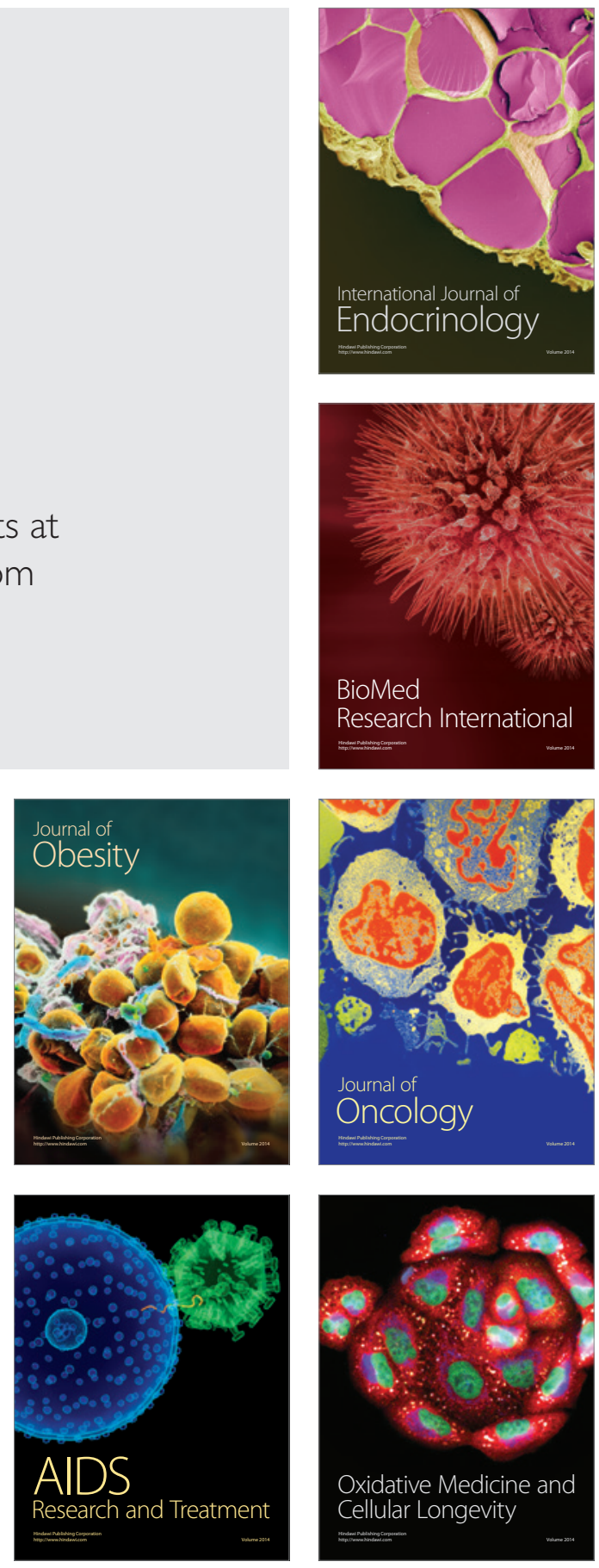